\title{
The Potential Use of Nanoparticles for Noggin siRNA Delivery to Accelerate Bone Formation in Distraction Osteogenesis
}

\author{
Ana Cristina Ferreira Bassit ${ }^{1,2 *}$, Pierre Moffatt ${ }^{1,3}$, Marie-Hélène Gaumond ${ }^{1}$ and Reggie Hamdy ${ }^{1,2}$ \\ ${ }^{1}$ Shriners Hospital for Children, Montreal, Quebec, Canada \\ ${ }^{2}$ Department of Experimental Surgery, McGill University, Montreal, Quebec, Canada \\ ${ }^{3}$ Department of Human Genetics, McGill University, Montreal, Quebec, Canada
}

\begin{abstract}
In bone research and orthopedics, RNA-interference (RNAi) and nanotechnology can be applied to a vast array of conditions where bone formation needs to be enhanced, providing new options to treat old problems, from osteoporosis and bone tumors to nonunion and critical size defects. The need for new therapeutic tools to improve bone regeneration is evident, mainly in more complex orthopedic surgical procedures. Distraction Osteogenesis (DO) is a unique surgical technique that stimulates bone formation, most frequently used to promote limb lengthening through slow and progressive distraction after osteotomy. The great challenge is to reduce the consolidation phase and prevent the complications related to the maintenance of the external fixator for a long period. Bone Morphogenetic Proteins (BMPs) have been used as potent osteoinductive growth factors, but we considered manipulating the BMP-antagonist NOGGIN through RNA silencing to increase endogenous BMPs levels, as an option for the controversial use of exogenous BMPs. For RNAi-based therapies, the greatest challenge over the past 16 years has been to achieve safe, effective and specific delivery to the target tissue. In-vivo studies employed a variety of delivery methods aiming for the ultimate clinical translational application for RNAi technology, but RNAi drugs are still undergoing clinical trials for approval by the US Food and Drug Administration (FDA). This review considers the application of nanotechnology to potentially address the difficulties of delivering silencing RNAs in vivo and in clinical applications, and presents preliminary studies about downregulation of the BMP-antagonist NOGGIN through RNAi in rat bone cells and delivery of siRNA sequences to bone tissue by nanoparticles.
\end{abstract}

Keywords: Nanoparticles; Distraction osteogenesis; RNA-silencing; RNA-interference; BMP; Noggin; Bone regeneration

\section{Introduction}

Nanotechnology, a term derived from the Greek prefix "nano"meaning dwarf, is the manufacturing of substances at the nanometer scale (one-billionth of a meter) or at the molecular level. Richard Feynman's lecture at the American Physical Society meeting in 1959, entitled "There is plenty of room at the bottom", is considered a landmark in nanotechnology, for bringing up the manipulation of atoms and manufacturing of nanoscale-sized structures [1-3]. Since its rise as a multidisciplinary scientific field, comprehending physics, chemistry, and biology, it quickly expanded to a multitude of applications in engineering and robotics, electronics and information technologies, medicine and genomics, agriculture and environmental control, among a vast list of research areas. But more than improving existing technologies, the applications in medical sciences created revolutionary new approaches on diagnostics and therapeutics by incorporating the advances from micro and nanotechnology into biotechnology, having as a major example the use of advanced micro arrays techniques in the Human Genome Project $[1,2,4]$.

Nanomedicine, or the application of nanotechnology to medical research and development of diagnostic tools and clinical treatments, exponentially evolved in the last decades. Improvements with the use of nanoparticles in noninvasive imaging techniques granted the opportunity of screening and monitoring intracellular changes by tagging biomolecules with quantum dots or synthetic chromophores. In molecular diagnostics, DNA flow through nanopores and incorporation of gold nanoparticles to short segments of DNA have been used for DNA sequencing. Nanotechnology promoted advances of great impact toward the development of new drugs and gene therapy, as nanoparticles can be taken up by cells, translocate to the cell cytoplasm and deliver small molecules, oligonucleotides and plasmid
DNAs, while protecting them from degradation and, at the same time, being potentially less immunogenic than viral vectors $[1,2,4]$.

The recent progress, and the application of nanotechnology to medicine and therapeutics, also offered new strategies to overcome drug delivery limitations, such as high toxicity, poor solubility, nonspecificity, degradation and short half-life after administration. Lipid-based nanoparticles proved to be effective to load small molecules, chemotherapeutic and antimicrobial drugs, and the use of liposomes was approved by the FDA for the delivery of doxorubicin and amphotericin B [5-8]. The acquired experience on the development of these compounds showed that they also have the potential to address the challenges of delivering silencing RNAs in vivo and in clinical applications.

RNA interference (RNAi) or gene silencing emerged with the potential to become one of the greatest therapeutic tools, with applications in different fields of medicine and for the treatment of a number of diseases, ranging from cancer, genetic disorders, viral infections, and autoimmune diseases [7-9]. In order to achieve such ambitious goals, RNAi had to evolve from the promising initial in

*Corresponding author: Ana Cristina Ferreira Bassit, Shriners Hospital for Children, Montreal, Quebec, Canada, Tel: 514-282-7165; E-mail: anafbassit@gmail.com

Received November 13, 2014; Accepted December 20, 2014; Published January 01, 2015

Citation: Bassit ACF, Moffatt P, Gaumond MH, Hamdy R (2015) The Potentia Use of Nanoparticles for Noggin siRNA Delivery to Accelerate Bone Formation in Distraction Osteogenesis. J Nanomed Nanotechnol 6: 257. doi: 10.4172/21577439.1000257

Copyright: @ 2015 Bassit ACF, et al. This is an open-access article distributed under the terms of the Creative Commons Attribution License, which permits unrestricted use, distribution, and reproduction in any medium, provided the original author and source are credited. 
vitro studies to safe and specific delivery of silencing sequences in vivo, avoiding off target effects and adverse immune reactions at the same time. Biotechnology and pharmaceutical companies have strongly invested on the development of RNAi drugs, but these agents are still being investigated and undergoing clinical trials for approval by the US Food and Drug Administration (FDA) [10,11]. During the past 16 years researchers had directed effort and resources to overcome RNAibased therapy's greatest challenge, which is to achieve safe, effective and specific delivery to the target tissue.

In bone research and orthopedics, nanotechnology and RNAi can be applied to a vast array of conditions where bone formation needs to be enhanced, providing new options to treat old problems, from osteoporosis and bone tumors to nonunion and critical size defects. The need for new therapeutic tools to improve bone regeneration is evident, mainly in more complex orthopedic surgical procedures as distraction osteogenesis, which final goal is to promote lengthening of the bone, increasing its total volume and mass through mechanical stimulation during distraction.

\section{RNA Interference and Nanoparticles as Delivery System}

Since Fire et al. identified double-strand RNAs (dsRNAs) and demonstrated RNA interference in C. elegans in 1998, it became the most studied method to attempt modifications in gene expression [12]. The long dsRNAs that mediate interference are processed by the Dicer enzyme into small or short interfering RNAs (siRNAs) duplexes containing 21-23 nucleotides that specifically target homologous mRNAs and promote their cleavage once incorporated into Argonaute 2 (AGO2) and the multicomponent RNA-induced silencing complex (RISC), blocking gene expression post-transcriptionally [13]. RNAi is a potent mechanism to promote functional gene inhibition without acting directly on the gene structure, that is highly conserved almost across all phyla. However, after it was demonstrated that siRNAs could also suppress gene expression in different mammalian cell lines, including human embryonic kidney 293 and HeLa cells [14,15], RNAi research and the use of chemically synthesized siRNAs quickly advanced toward in vivo use of siRNAs and their therapeutic applications. Since then, with the development of more advanced algorithm software, use of automated neural networks and support vector machines to select the sequences, siRNA design has greatly improved silencing potency.

Nevertheless, the use of RNAi in vitro is a well-established research tool, while efficient and safe delivery of siRNAs and shRNAs remains as the greatest challenge for the development of in vivo methods and subsequent use of RNAi based therapies [10,11]. Many of these therapies were approved for clinical trial by the FDA; until 2011, approximately 30 clinical trials were investigating the use of RNAi technology, particularly for disease conditions with validated genetic targets that are difficult to be addressed with the current available drug armamentarium. Many failed or had to be terminated for not achieving the objectives or for showing strong adverse effects [11]. At a moment of disappointments in the scientific community and huge monetary losses, the use of nanoparticles emerged as a rescuing alternative for siRNA delivery in vivo.

Nanoparticles are structures of sizes ranging from 1 to $100 \mathrm{~nm}$ in at least one dimension, according to the definition from NNI (National Nanotechnology Initiative). One of the major advantages of nanosized particles, including both polymer- and lipid-based systems, is that they can serve as vehicles for many therapeutic applications, providing targeted drug delivery to the site of disease and avoiding undesirable side effects. However, to be used as therapeutic tools, lipid nanoparticles
(LNPs) must have long half-lives to circulate in the blood for a long period and be able to accumulate in the target tissue. If not modified, LNPs are labeled as foreign substances aggravating the immune system through the adsorption of opsonins (plasma proteins) such as immunoglobulins or complement fragments to their surface, attacked by monocytes and neutrophils, and cleared by the reticuloendothelial system. In order to avoid this opsonization and increase their half-life, LNPs are usually coated with a neutral hydrophilic surface layer, a process known as stealth coating, most commonly obtained through "PEGylation" - layering with polyethylene glycol(PEG), a biocompatible and inert polymer - to provide surface stabilization. Many formulations combining PEGylated liposomes and chemotherapeutic drugs have been already approved and used against a variety of tumors (Kaposi's sarcoma, ovarian cancer, multiple myeloma) [5,7]. Poly (amino acids), as well as other alternative structures, have been developed with the same stealth function, protecting LNPs and prolonging their systemic half-life, but also exhibiting reduced risk of accumulation and related toxicity, as they are readily degraded by proteases $[7,11,16,17]$.

The improvements obtained in drug delivery with lipid-based systems encouraged the development of liposomal siRNA delivery systems as well. The evolution of nanotechnology and nanomedicine came to offer alternative siRNA delivery strategies to overcome the limitations of "naked" siRNAs, such as the rapid degradation in biological fluids and difficulties to cross the cell membrane and accumulate in the target tissues. Nanoparticles can also help to overcome nuclease degradation, provide more stability to the constructs, reduce toxicity, and avoid off target effects such as innate immune responses and unintended suppression of non targeted RNAs. For these purposes, lipid or liposomal nanoparticles (LNPs) containing ionizable amino lipids are synthesized in the 100-nm size range and represent the most common system used for siRNA in vivo delivery and RNAi-based therapeutics, mainly for systemic applications. Lipidbased siRNA are versatile carriers and the majority also bears a cationic charge to facilitate the passage of siRNA across cell membrane barriers. siRNAs directly formulated in liposomes are available from various commercial sources and promising clinical results have been achieved using this new class of therapeutics [7,11,17]. A large number of studies in animal models have shown that LNPs are capable to deliver siRNA via intravenous injections and lead to gene silencing in many different target tissues and disease conditions [11,18]. RNAi was achieved in humans by Davis et al. who demonstrated, in a Phase I clinical trial, the first example of dose dependent accumulation using targeted nanoparticles to deliver siRNA into metastatic melanomas [19]. This consisted in a great advantage over the delivery by lentiviral vectors, not yet approved for clinical applications of RNAi.

Lentiviral vectors, derived from feline (FTV) and human immunodeficiency virus (HIV)-1, were developed because of their ability to transduce cells that proliferate poorly or not at all; they can infect both dividing and nondividing cells, providing long-term transgenic expression once their genetic cargo is integrated into the target cell chromosome. Once integrated to the host genome, they allow for stable gene delivery, and can be used to generate transgenic animals that exhibit loss-of-function phenotypes, besides vector transmission to offspring [20].

Many improvements have been gradually introduced to increase lentiviruses' levels of versatility, efficiency and biosafety, guaranteeing that the only information that is transferred to the target cells is the genetic information contained in the vector genome. Lentiviruses are not encapsulated and do not transfer sequences that encode for 
proteins derived from the packaging virus, reducing the risk of being attacked by lymphocytes, what make them less immunogenic. In addition, when generated by three different plasmids (for the genomic RNA, the internal structural and enzymatic proteins, and the envelope glycoprotein), the vector particles produced are replication-defective viruses, allowing experimental studies to be conducted with lower risks - biosafety level 2 (BSL-2). They also can be concentrated to high titers, exhibit great tropism and a relatively large capacity (approximately $10 \mathrm{~kb}$ ), allowing the delivery of most cDNAs. The combination of all these interesting features brings lentiviral vectors to high consideration as an attractive and powerful gene delivery system in research [21,22], although not as successful for in vivo applications; the differences between the main two delivery systems for RNAi are represented in Figure 1 .

\section{RNAi and Nanotechnology in Bone Research and Orthopedics}

Bone has always represented a difficult target for drug delivery systems. Until this date, the intermittent administration of parathyroid hormone (PTH) is the only bone anabolic treatment approved for osteoporosis cases associated with severe impairment of bone formation. Despite its static appearance, bone is a dynamic tissue going through constant modeling and remodeling throughout life, in a coordinated process of bone resorption followed by bone formation. Differently from soft tissues that generate scar tissue when injured, bone regenerates by forming new tissue during fracture healing, recapitulating the embryonic steps of osteogenesis, with similar recruitment of cells and activation of molecular signaling pathways, enabling complete restoration of its structural architecture. However, when injuries caused by trauma, tumors, malformations or complications from previous surgical procedures are extensive, causing severe bone loss, the current treatment modalities fail to support the bone healing process until full recovery is achieved. In these cases, additional treatment strategies, as grafting, Ilizarov ring external fixation, distraction osteogenesis, use of

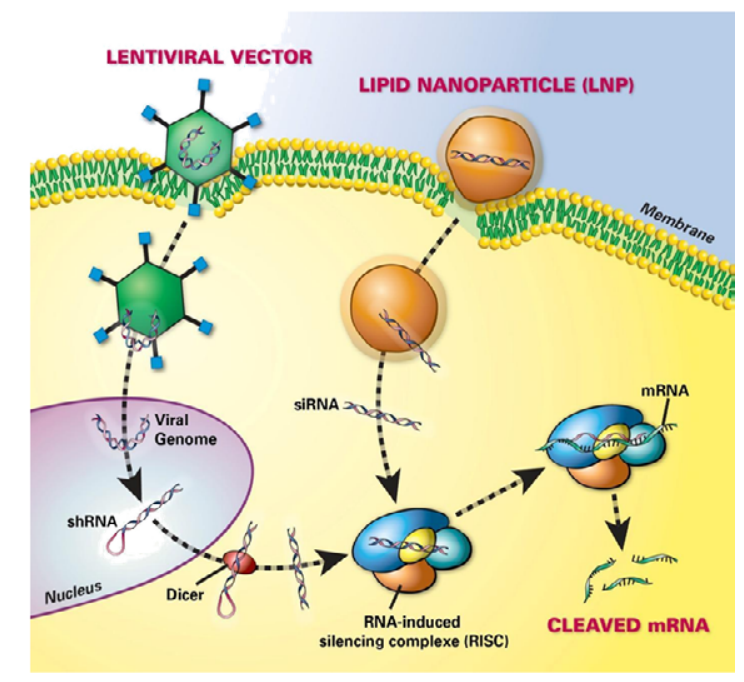

Figure 1: The two main delivery systems for RNAi: lipid-based nanoparticles and lentiviral vectors. Synthetic siRNA can be carried by lipid nanoparticles into the cytoplasm, where they are incorporated to the RNA-inducing silencing complex (RISC), which targets and degrades the complementary messenger RNA. Lentiviruses deliver the plasmid into the nucleus for transcription, and the shRNA sequences are then exported to the cytoplasm, where they are cleaved by the enzyme Dicer into siRNA duplexes before combining with RISC

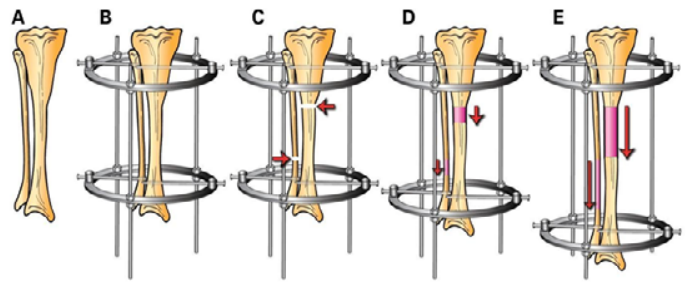

Figure 2: Steps of the technique of distraction osteogenesis used for limb lengthening; A. Intact bone before the surgical procedure; B. Application of the external fixator; C. Osteotomy sites; D. Start of distraction, and E. End of distraction when the desired amount of lengthening is achieved.

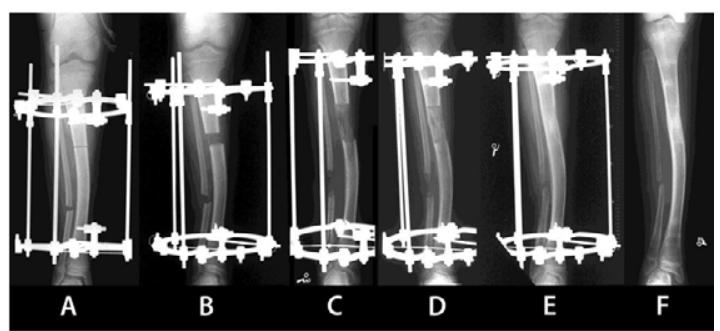

Figure 3: Radiological representation of the distraction osteogenesis technique. A. Osteotomy is performed after the application of the external fixator; B. Initial phase of the distraction procedure. C. Elongation of the bone; D. New bone tissue formed at the distraction site; E. Radiological evidence of bone mineralization during the consolidation phase; F. Final consolidation of the lengthened bone and removal of the fixator.

growth factors, and therapeutic electric devices, must be introduced. Fracture non-unions increase patients' morbidity and mortality, represent a difficult challenge for the surgeon and an economic burden for the health systems and social services, mainly with the rising of high energy trauma rates and an ageing population.

Distraction osteogenesis (DO) is a unique surgical technique, used to promote limb lengthening, which stimulates bone formation through slow and progressive distraction. It is widely used for the treatment of limb length discrepancies, limb deformities, long bone non-unions, and bone loss secondary to infection, trauma and malignancies. The surgical procedure consists of the application of an external fixator device to the affected bone, followed by a low-energy transverse osteotomy and gradual distraction of the two bone segments (Figures 2 and 3). The formation of new bone tissue within the distracted gap occurs in three phases: latency, lengthening or distraction, and consolidation phase $[23,24]$.

During the latency phase, which lasts 5 to 7 days, no distraction maneuver is performed, avoiding any interference with the ongoing initial inflammatory response, involving migration of pluripotential cells and secretion of growth factors and cytokines. During the subsequent lengthening or distraction phase, the bone segments are gradually distracted till obtaining the desired total bone length. The appropriate mechanical tension-stress can stimulate and maintain bone formation. The optimum amount of daily lengthening is $1.0 \mathrm{~mm}$, divided into 2 to 4 increments, as the distraction rate should guarantee that the surrounding tissues can adapt without damage to nerves, blood vessels, muscle and skin. The consolidation phase is the period that the fixator has to be kept in place until the new bone tissue generated in the distraction gap is strong enough to withstand the physiological mechanical load [25]. 
However, the consolidation phase is long and the maintenance of the external fixator for such an extensive period is commonly associated with unfavorable outcomes due to post-operative complications, such as pin site infections that range from simple discharge to osteomyelitis, edema of the lengthened limb, intense musculoskeletal pain and the possibility of repeated surgical interventions. The delay of bone formation, leading to psychological and medical complications, has a huge financial impact on the patient as well as on the health care system. The great challenge is to accelerate bone formation reducing the consolidation phase, that lasts about one month for every $\mathrm{cm}$ lengthened, and prevent the complications related to the maintenance of the external fixator for a long period.

Nanoparticles have potential applications in bone tissue engineering and regeneration, exhibiting approximately the same size range as natural components of bone, such as the hexagonal crystal structures of hydroxyapatite and major organic components as collagen fibrils, and can be integrated to organic and mineral constituents of bone, as well as function as $2 \mathrm{D}$ or $3 \mathrm{D}$ nanostructured scaffolds for bone regeneration, or vehicles for delivery of drugs and genetic material $[2,3,26]$. The integrity of bone structure and skeletal homeostasis are maintained through constant bone turnover that takes place in the basic multicellular unit (BMU), which contains osteoclasts and osteoblasts, cells involved in bone resorption and bone formation respectively, and osteocytes, former osteoblasts that became trapped in the secreted and then mineralized matrix. These cells within the bone-remodeling cavity are protected by a canopy of bone-lining cells, possess capillary supply, and their coupled action in bone turnover occurs through an array of signaling pathways that tightly coordinates bone resorption and formation [27-30].

It was in this niche that Zhang et al. demonstrated the strong affinity of a targeting oligonucleotide - (AspSerSer) to bind bone-formation surfaces predominantly with osteoblasts, that are characterized by lowly crystallized hydroxyapatite, differently from osteoclast predominant bone-resorption surfaces, characterized by highly crystallized hydroxyapatite $[31,32]$. In the sequence, the (AspSerSer) targeting moiety was linked with a DOTAP-based cationic liposome (FDA approved), encapsulating an osteogenic siRNA targeting a negative regulator (Plekho1) of osteogenic lineage activity without modulating bone resorption. After tail injection, the (AspSerSer) liposome with the Plekho1 siRNA showed strong fluorescent signal in trabecular bone and weak signal in other organs compared to liposomes without (AspSerSer) ${ }_{6}$ that contained Plekho1 siRNA. Most importantly, in vivo results showed efficient knockdown of the Plekho1 gene in bone after treatment with (AspSerSer)-liposome plus Plekho1 siRNA as compared to the knockdown achieved by treatment with the liposome (without (AspSerSer) ${ }_{6}$ ) plus Plekho1. In addition, the siRNA administration of (AspSerSer) -liposome plus Plekho1 siRNA resulted in increased bone mass and improved trabecular architecture. RNAimediated bone anabolic action in osteoporotic rats was also confirmed by treating ovariectomized female rats with (AspSerSer) ${ }_{6}$ containing Plekhol siRNA and achieving restoration of bone micro-architecture. In both healthy and osteoporotic rats, the gene knockdown occurred in a bone-selective manner, as bone resorption was not activated by (AspSerSer) ${ }_{6}$-liposome plus Plekho1 siRNA [32].

Nanomedicine also showed to be an important alternative for the challenging treatment of tumors within the bone microenvironment. Nanoparticles consisting of poly glycolic acid (PLGA), polyethylene glycol (PEG), and bisphosphonate (alendronate) were used to provide bone targeted delivery of bortezomib, a model drug for the treatment of multiple myeloma, a plasma cell cancer that proliferates primarily in bone marrow and causes osteolytic lesions [33].

\section{Targeting Bone Regeneration during DO}

Several methods have been developed to stimulate bone formation in DO, including the local application of growth factors, especially Bone Morphogenetic Proteins (BMPs) [23,25,34,35], members of the transforming growth factor (TGF)- $\beta$ superfamily of signaling molecules, which participate in the embryonic development of many tissues, mainly in the skeletal system. Amongst other growth factors, BMPs (mainly BMP-2, -4 and -7) play a key role in bone healing, inducing the differentiation of mesenchymal stem cells into cells of the osteoblastic lineages, as well as stimulating osteogenic activity in mature osteoblasts [36-39].

BMPs interact with specific cell-surface receptors (BMPR-I and BMPR-II), resulting in mobilization of members of the Smad family, Smads 1-, 5, and -8. Once these signal transducing molecules are phosphorilated, they bind to Smad- 4 and then translocate to the nucleus, where this new formed complex binds to specific DNA binding proteins. These proteins act as co-activators with higher affinity for the response element sequences in DNA, regulating the expression of BMP target genes. There is also an alternative pathway intermediated by MAP kinases to modulate the transcriptional activity [40-43].

Since their discovery by Urist in 1965, and the innumerous in vitro and in vivo studies that followed [39,44-47], BMPs have been considered potent osteoinductive growth factors, able to enhance bone formation and accelerate fracture consolidation in several conditions where bone repair is impaired [48-51]. Meta-analysis of results from BMPs' clinical trials indicated better rates of bone healing, compared to the gold standard treatment with autologous bone grafts, when considering clinical and radiological outcomes [28]. When focusing on $\mathrm{DO}$, the mechanical tension-stress generated during the distraction has a marked effect on gene and protein expression of BMPs. The mRNA levels of BMP-2 and -4, and protein expression of BMP-2, -4 and -7 are detected after osteotomy, but instead of showing a gradual decrease as in normal fracture repair, they increase as distraction is initiated $[35,52,53]$. In human fractures and non-unions tissue samples, coexpression of BMPs and BMP-inhibitors was observed, although the expression of BMP inhibitors (noggin, gremlin, chordin, Smad-6, Smad-7 and BAMBI) was higher. In addition, the expression of BMP-2 was decreased and the expression of BMP-7 was absent in hypertrophic and non-hypertrophic chondrocytes, revealing an imbalance BMPs and BMP inhibitors in non-unions compared to normal healing fractures [54]. The recombinant forms of BMP-2 and -7 are approved by the FDA to promote spinal fusion and fracture healing [27].

However, due to the rapid clearance of BMPs from the circulation when locally applied, large supra-physiological doses have to be administered in the clinical setting to accelerate bone growth. These large doses may have significant short and long term complications, notwithstanding the elevated costs associated with the use of recombinant human BMPs (rhBMPs). Another therapeutic strategy to be considered is to increase the local levels of endogenous BMPs by inhibiting BMP antagonists, and promote enhanced osteogenesis. The biological activities and mechanisms for signaling transduction of BMPs are regulated by a variety of intracellular and extracellular antagonists, that are equally important for neuronal and body axis development in the embryo [37,42,43,55].

NOGGIN, a $64 \mathrm{kDa}$ glycoprotein, is a major BMP extracellular 
antagonist, closely related to the osteoinductive action and anabolic effects of BMPs. It is a ligand-binding type of antagonist that directly binds to BMPs, with different affinity and specificity levels to BMPs-2, $-4,-5,-6$ and -7 , preventing their interaction with receptors BMPR-I and -II on the cell surface and the subsequent activation of the BMP pathway (Figure 4). NOGGIN is also produced and secreted by osteoblasts, and its expression is essential for adequate skeletal and joint development. Excessive bone and cartilage formation, with thickened long bones, and joint deformities occur in Noggin knockout mice [56]. On the other hand, transgenic mice overexpressing NOGGIN in mature osteoblasts under the control of mouse osteocalcin promoter exhibited significant osteopenia and long bone fractures in the first month of life, related to an accentuated decrease in bone mineral density, trabecular bone volume and trabecular number, and bone formation rates [57,58]. Several in vitro studies, mostly in cells of the osteoblast lineage, demonstrated that NOGGIN administration inhibits BMP signaling, with downregulation of BMP bone anabolic effects [58-61].

However, the most promising results were obtained from the studies developed by Wan et al. in 2007 (41), introducing NOGGIN downregulation through RNA interference to manipulate the osteogenic activity of BMPs. Noggin suppression in MC3T3-E1 pre-osteoblasts, via small-interfering RNA (siRNA) constructs, showed marked effects on bone formation, increasing SMADs 1 and 5 expression and BMP signaling. In vivo, mice with critical size calvarial defects were treated with siRNA infected primary osteoblasts and demonstrated accelerated repair. Increased bone formation was demonstrated by histological analysis and radiographical analyses post-operatively [41]. These results strongly suggest that NOGGIN downregulation through RNA interference can be used to modulate BMP activity and increase osteogenesis in the early stages of fracture healing. Based upon these data, we decided to evaluate the efficacy of shRNA sequences to silence or downregulate Noggin expression in vitro and in vivo, as well as the use of nanoparticles for siRNA delivery to bone cells.

\section{The Potential Use of RNAi to Downregulate the BMP- Antagonist NOGGIN: Preliminary Experimental Results}

As previously mentioned, BMPs have been used as potent growth factors, but we considered manipulating the BMP-antagonist NOGGIN

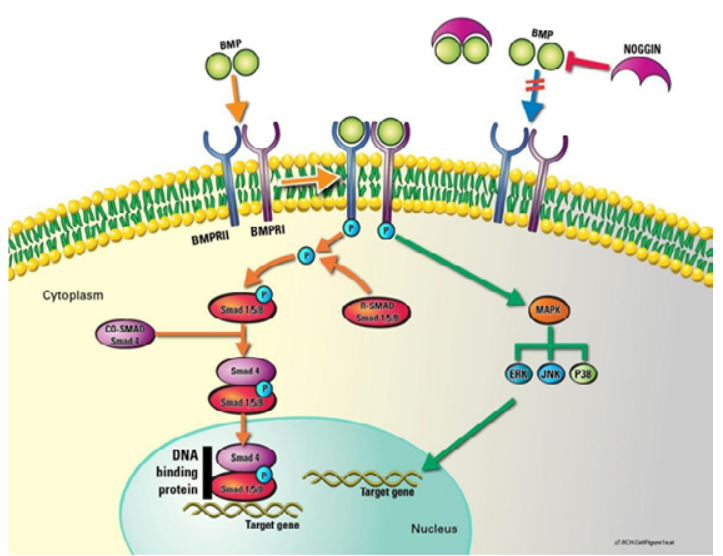

Figure 4: BMP inhibition by the antagonist NOGGIN. BMPs bind to receptors I and II (BMPR-I and BMPR-II) at the cell surface leading to phosphorilation of Smads 1,5 and 8. After binding to Smad 4 the complex is translocated to the nucleus, to promote gene transcriptional activation. There is also an alternative pathway through MAP kinases. NOGGIN acts upstream in this pathway, preventing binding of BMPs to the receptors. through RNA silencing to increase the endogenous BMPs levels, as an option for the controversial use of exogenous BMPs to accelerate bone formation in DO. In a previous study, shRNA constructs targeting Noggin were able to promote downregulation of NOGGIN in MC3T3-E1 preosteoblasts and primary mouse calvarial osteoblasts, with enhanced expression of osteogenic differentiation markers and bone deposition, as well as increased expression of SMAD- 1 and -5 in the infected cells compared to sham infected cells. In addition, in vivo critical-sized calvarial defects showed accelerated healing at 2 and 4 weeks after injury [41].

We first analyzed the expression profile of the Noggin gene in growing and differentiating bone cells. Our kinetic study, with vector based short hairpin RNA (shRNA) and lentiviruses, confirmed that Noggin is constitutively expressed in differentiating MC3T3-E1 osteoblasts and peak in expression before the onset of mineralization. Differently from siRNAs, that are cleaved by Dicer or loaded directly to RISC in the cytoplasm, shRNAs are delivered by expression vectors that have to be translocated into the nucleus for subsequent transcription. Following transcription, the shRNA sequence is exported to the cytoplasm, where it is recognized and cleaved at the loop by Dicer, which processes the shRNA into siRNA duplexes. From this step on, like the naturally occurring or exogenous synthetic siRNAs, the shRNA-derived siRNAs enter the silencing pathway through incorporation to RISC complex for target-specific mRNA degradation [13,62-64]. We have also used lentiviruses previously and showed that they can infect osteoblasts with high efficiency in vitro [65], and more recently we have had success with lentiviral vectors as delivery system for shRNA-mediated RNA interference in MC3T3 cells (unpublished data). Overall our results indicated that we can robustly knockdown NOGGIN proteins in our in vitro osteoblastic model using shRNA-expressing lentiviral particles; and further, that this knockdown has a significantly positive effect on BMP signaling pathway, revealed by increase in alkaline phosphatase (ALP) levels, a marker of the osteogenic differentiation, which expression is influenced by BMP- 2 through the $\mathrm{Wnt} / \beta$-catenin signaling [66]. The ALP assays were performed on day 2 and day 4, on cell cultures stably expressing the shRNA sequence targeting Noggin. They were compared to the control NT. The results showed an increase of the ALP activity that was significant for Noggin at both time points.

The rat was considered a more adequate animal model, as its size and body weight (reaching 450 to $600 \mathrm{~g}$ ) would provide a better surgical access to perform the DO surgeries, as well as allowing larger volumes of the substances to be injected at the distraction site. Therefore, we decided to evaluate Noggin silencing in rat osteoblastic cells (UMR-106 rat osteosarcoma cells). As a first approach to evaluate the NOGGIN downregulation, we conducted an in vitro study to compare the efficacy of five different short hairpin RNA (shRNA) sequences targeting Noggin, designed and produced by Sigma Mission ${ }^{\circledR}$-shRNA. They encoded for 20-21 nucleotides matching 5 different specific sequences of the mouse (NM_008711) and rat (NM_012990) Noggin cDNAs (denoted by the base location on the cDNA) (Figure 5).

The 20-mer shRNA sequences were cloned downstream of the U6 promoter of the lentiviral plasmid pLKO.1 puro - Sigma ${ }^{\circ}$. A nontarget shRNA (pLKO.1-NT; Sigma) was used as a negative control. These vectors also encoded a drug resistance gene inactivating puromycin for posterior selection of the cells (Figure 6).

The production of lentiviruses expressing the shRNAs was achieved by transfection of HEK (Human Embryonic Kidney 293) cells, grown in DMEM (Dulbecco's modified Eagle's medium) supplemented with $10 \%$ FBS, with X-tremeGene 9 DNA Transfection Reagent - Roche ${ }^{\circ}$. 
Citation: Bassit ACF, Moffatt P, Gaumond MH, Hamdy R (2015) The Potential Use of Nanoparticles for Noggin siRNA Delivery to Accelerate Bone Formation in Distraction Osteogenesis. J Nanomed Nanotechnol 6: 257. doi: 10.4172/2157-7439.1000257

noggin NM 008711 (699bp)

Figure 5: Position of Noggin shRNAs in cDNA: \#1 - (165-185) shRNA N185, \#2 - (420-440) shRNA - N420, \#3 - (137-157) shRNA - N157, \#4 - (78-98) shRNA - N98, \#5 - (354-374) shRNA - N374.

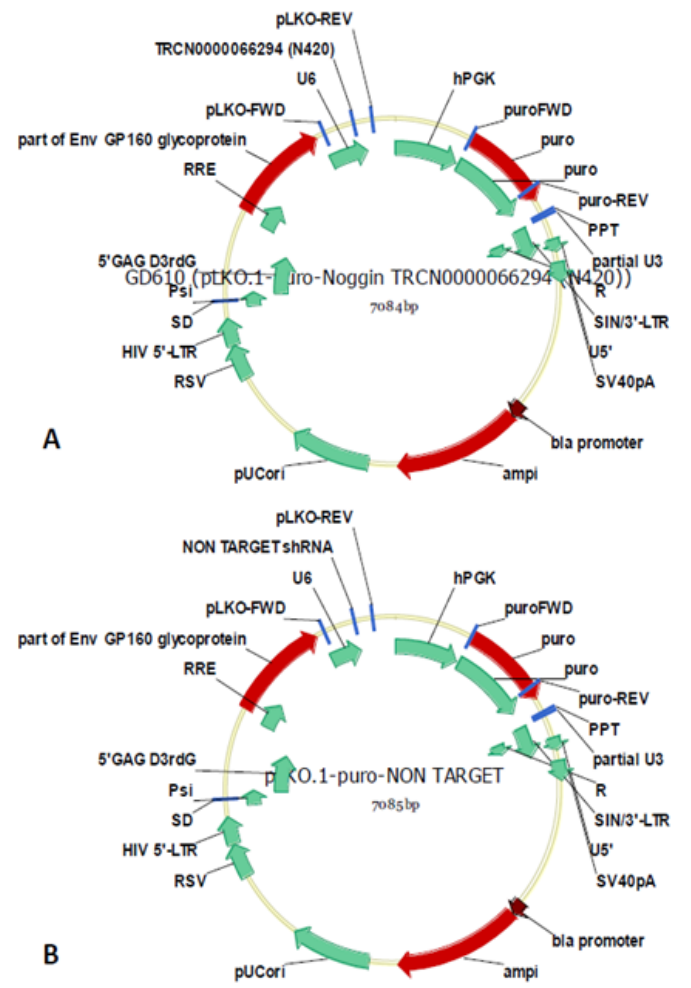

Figure 6: Lentiviral plasmids encoding shRNA sequence targeting Noggin (A) and the non-target sequence (B). Both vectors also included puromycin

Seventy two hours after transfection, the media was collected and lentiviral particles were concentrated by ultracentrifugation. UMR106 rat osteosarcoma cells were seeded in 12-well plates and infected with the Noggin and nontarget shRNA lentiviruses. Following amplification and selection with puromycin, the cells were seeded in two 12-well plates, at 300,000 cells/well, for RNA and protein extraction. Cells and conditioned media were collected and processed for qRT-PCR and Western blotting to analyze Noggin mRNA expression and protein production and secretion.

UMR cells infected with lentiviruses expressing shRNA N185 and N420 showed $42 \%$ and $45 \%$ decrease of Noggin gene expression as assessed by qRT-PCR (Figure 7). The Western blot analysis, using $12 \%$ SDS-PAGE gels, showed that NOGGIN protein migrated at $\sim 25 \mathrm{kDa}$. Total cell lysate and conditioned medium collected at the experimental end point revealed that NOGGIN was downregulated to qualitatively less than half and less than one fifth as compared to the control in cells infected with shRNA N185 and N420 respectively (Figure 8).

The use of shRNA against the BMP-antagonist NOGGIN proved to be effective in vitro, promoting significant decrease of RNA and protein levels of osteoblastic cell lines infected by lentiviral vectors, yet to be
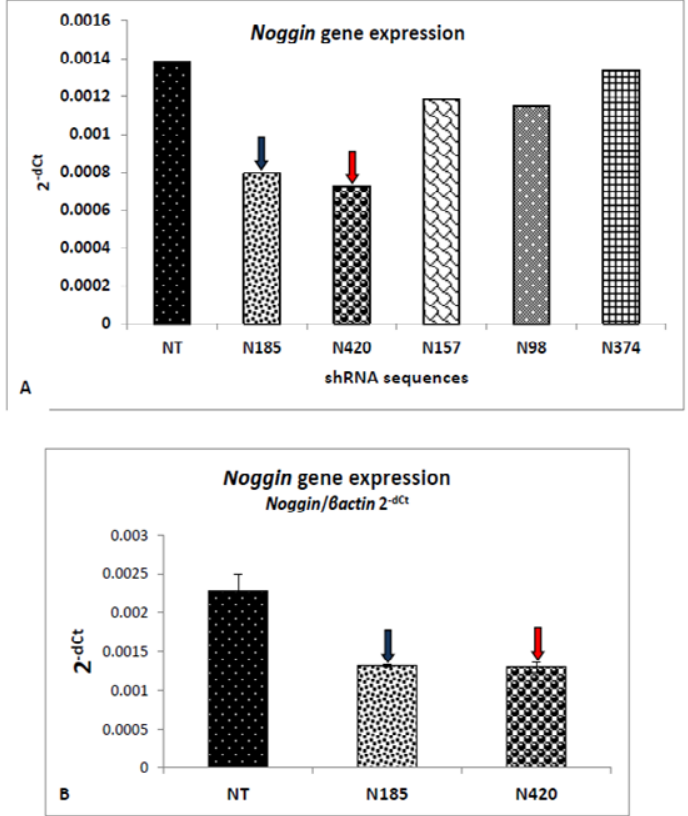

Figure 7: Noggin mRNA gene expression, measured by qRT-PCR and with values normalized to $\beta$ actin, showed $42 \%$ and $45 \%$ decrease in cells infected with lentiviruses delivering shRNA sequences N185 and N420, among the five shRNA sequences analyzed $(A)$, and when just compared to the non target control $(B)$.
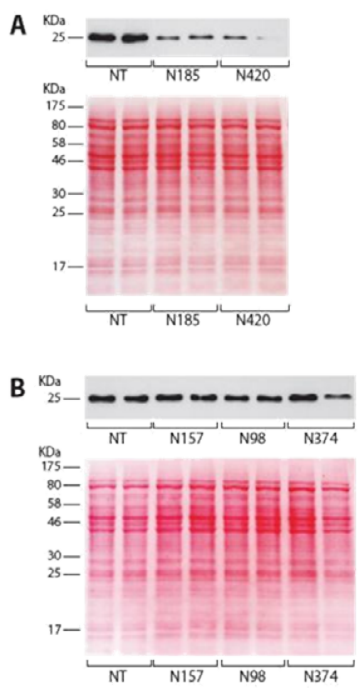

Figure 8: Western blot detection of NOGGIN in UMR106 cells processed and collected for analysis in $12 \%$ SDS PAGE. Rat NOGGIN migrated as a single band consistent with its predicted mass of $25 \mathrm{KDa}$, and showed decreased expression in cells infected with shRNA N185 and N420.

tested in vivo, with rats as animal models.

\section{The Potential Use of Nanoparticles as Delivery System For Pten siRNA to Bone Cells: Preliminary Results}

In our study involving nanoparticles we used 1-palmitoyl, 2-oleoyl phosphatidylcholine, cholesterol and the triglyceride triolein nanoparticles in the $20-\mathrm{nm}$ range to target extravascular tissues, produced through integrating microfluidics technology 
Citation: Bassit ACF, Moffatt P, Gaumond MH, Hamdy R (2015) The Potential Use of Nanoparticles for Noggin siRNA Delivery to Accelerate Bone Formation in Distraction Osteogenesis. J Nanomed Nanotechnol 6: 257. doi: 10.4172/2157-7439.1000257

Page 7 of 8

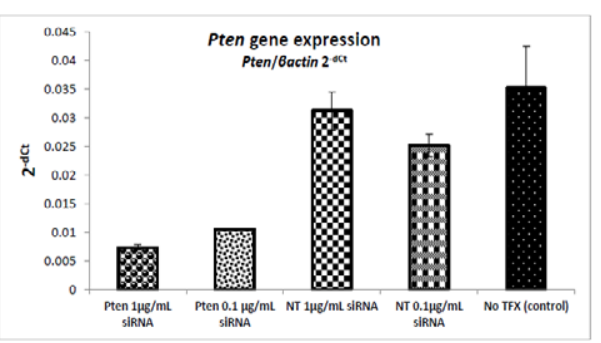

Figure 9: Pten mRNA gene expression, as measured by qRT-PCR and with values normalized to $\beta$ actin, showed $71 \%$ and $89 \%$ downregulation in cells transfected with lipid nanoparticles delivering siRNA sequences at 0.1 and $1 \mu \mathrm{g} / \mathrm{mL}$ concentration.

$[67,68]$ by Precision Nanosystems ${ }^{\bullet}$. It was demonstrated that these nanoparticles, combined with siRNA sequence targeting Pten gene (phosphatase and tensin homolog gene), could induce $85 \%$ of Pten gene knockdown in primary neurons culture at $10 \mathrm{ng} / \mathrm{mL}$ siRNA, and sustained $80 \%$ gene knockdown in vivo, for approximately 15 days after intracerebroventricular injection [67].

Pten is a tumor suppressor gene, and the protein PTEN is found in almost all tissues in the body. Our first goal was to evaluate the uptake of these nanoparticles by osteoblastic cells and the potential PTEN knockdown in bone tissue.

UMR-106 -rat osteosarcoma cells were grown in 24-well plates with DMEM (Dulbecco's modified Eagle's medium) supplemented with $10 \%$ FBS till reaching adequate confluence. The cells were then transfected with Pten RNAi nanoparticles and non-target (NT) RNAi nanoparticles in duplicates, at two different concentrations: 0.1 and $1 \mu \mathrm{g} / \mathrm{ml}$ siRNA. Non-infected cells were also kept as control, and incubation proceeded under normal cell culture conditions for 48 hours. Cells were collected and processed for qRT-PCR to analyze Pten mRNA expression.

Cells expressing siRNA targeting Pten, at both concentrations showed $71 \%$ and $89 \%$ decrease in gene expression when compared to nontransfected (control) cells by qRT-PCR analysis of UMR cells (Figure 9).

The use of siRNA against Pten proved to be effective in vitro, in rat osteoblastic cell lines, promoting significant decrease of mRNA expression in cells transfected with siRNA delivered by lipid nanoparticles. Nanoparticle-based delivery system is a promising alternative method for therapies using silencing RNAs in bone tissue, yet to be tested in vivo, and with potential application during DO.

Ongoing and future studies in our laboratory include the in vivo evaluation of Pten siRNA delivery by lipid-based nanoparticles to bone tissue after intra-osseous injection in rat femora. If confirmed, the same nanoparticle system would be tested to deliver the siRNA sequences targeting Noggin, focusing on its potential application to enhance bone regeneration during distraction osteogenesis surgeries.

\section{Acknowledgment}

The authors thank Guylaine Bedar and Mark Lepik for their collaboration with the illustrations on this article, and Euan Ramsay and Anitha Thomas, from Precision Nanosystems $₫$ for providing the nanoparticles.

\section{References}

1. Emerich DF, Thanos CG (2003) Nanotechnology and medicine. Expert Opin Biol Ther 3: 655-663.

2. Sahoo SK, Parveen S, Panda JJ (2007) The present and future of nanotechnology in human health care. Nanomedicine 3: 20-31.

3. Gusic N, Ivkovic A, VaFaye J, Vukasovic A, Ivkovic J, et al. (2014) Nanobiotechnology and bone regeneration: a mini-review. Int Orthop 38: 1877 1884

4. LaVan DA, Lynn DM, Langer R (2002) Moving smaller in drug discovery and delivery. Nature reviews Drug discovery 1: 77-84.

5. Parveen S, Misra R, Sahoo SK (2012) Nanoparticles: a boon to drug delivery, therapeutics, diagnostics and imaging. Nanomedicine 8: 147-166.

6. Rust DM, Jameson G (1998) The novel lipid delivery system of amphotericin B: drug profile and relevance to clinical practice. Oncol Nurs Forum 25: 35-48.

7. Wan C, Allen TM, Cullis PR (2013) Lipid nanoparticle delivery systems for siRNA-based therapeutics. Drug Delivery and Translational Research 4: 74-83.

8. Whitehead KA, Langer R, Anderson DG (2009) Knocking down barriers: advances in siRNA delivery. Nat Rev Drug Discov 8: 129-138.

9. Schroeder A, Levins CG, Cortez C, Langer R, Anderson DG (2010) Lipid-based nanotherapeutics for siRNA delivery. J Intern Med 267: 9-21.

10. Lee SH, Sinko PJ (2006) siRNA--getting the message out. Eur J Pharm Sc 27: 401-410.

11. Rettig GR, Behlke MA (2012) Progress toward in vivo use of siRNAs-II. Mo Ther 20: 483-512.

12. Fire A, Xu S, Montgomery MK, Kostas SA, Driver SE, et al. (1998) Potent and specific genetic interference by double-stranded RNA in Caenorhabditis elegans. Nature 391: 806-811.

13. Rao DD, Vorhies JS, Senzer N, Nemunaitis J (2009) siRNA vs. shRNA: similarities and differences. Adv Drug Deliv Rev 61: 746-759.

14. Elbashir SM, Harborth J, Lendeckel W, Yalcin A, Weber K, et al. (2001) Duplexes of 21-nucleotide RNAs mediate RNA interference in cultured mammalian cells. Nature 411: 494-498.

15. Elbashir SM, Harborth J, Weber K, Tuschl T (2002) Analysis of gene function in somatic mammalian cells using small interfering RNAs. Methods 26: 199-213.

16. Amoozgar Z, Yeo Y (2012) Recent advances in stealth coating of nanoparticle drug delivery systems. Wiley Interdiscip Rev Nanomed Nanobiotechnol 4: 219 233.

17. Li W, Szoka FC Jr (2007) Lipid-based nanoparticles for nucleic acid delivery. Pharm Res 24: 438-449.

18. Dong Y, Love KT, Dorkin JR, Sirirungruang S, Zhang Y, et al. (2014) Lipopeptide nanoparticles for potent and selective siRNA delivery in rodents and nonhuman primates. Proc Natl Acad Sci USA 111: 3955-3960.

19. Davis ME, Zuckerman JE, Choi CH, Seligson D, Tolcher A, et al. (2010) Evidence of RNAi in humans from systemically administered siRNA via targeted nanoparticles. Nature 464: 1067-1070.

20. Leung AK, Hafez IM, Baoukina S, Belliveau NM, Zhigaltsev IV, et al. (2012) Lipid Nanoparticles Containing siRNA Synthesized by Microfluidic Mixing Exhibit an Electron-Dense Nanostructured Core. J Phys Chem C Nanomater Interfaces 116: 18440-18450.

21. Barde I, Salmon P, Trono D (2010) Production and titration of lentiviral vectors. Curr Protoc Neurosci Chapter 4: Unit 4.

22. Pauwels K, Gijsbers R, Toelen J, Schambach A, Willard-Gallo K, et al. (2009) State-of-the-art lentiviral vectors for research use: risk assessment and biosafety recommendations. Curr Gene Ther 9: 459-474.

23. Hamdy RC, Rendon JS, Tabrizian M (2012) Distraction Osteogenesis and Its Challenges in Bone Regeneration, Intech.

24. llizarov GA (1989) The tension-stress effect on the genesis and growth of tissues: Part II. The influence of the rate and frequency of distraction. Clin Orthop Relat Res: 263-285.

25. Sabharwal S (2011) Enhancement of bone formation during distraction osteogenesis: pediatric applications. J Am Acad Orthop Surg 19: 101-111. 
Citation: Bassit ACF, Moffatt P, Gaumond MH, Hamdy R (2015) The Potential Use of Nanoparticles for Noggin siRNA Delivery to Accelerate Bone Formation in Distraction Osteogenesis. J Nanomed Nanotechnol 6: 257. doi: 10.4172/2157-7439.1000257

Page 8 of 8

26. Tautzenberger A, Kovtun A, Ignatius A (2012) Nanoparticles and their potential for application in bone. Int J Nanomedicine 7: 4545-4557.

27. Khosla S, Westendorf JJ, Oursler MJ (2008) Building bone to reverse osteoporosis and repair fractures. J Clin Invest 118: 421-428.

28. Lian JB, Stein GS (2006) The Cells of Bone. In: Dynamics of Bone and Cartilage Metabolism.

29. Rosen CJ (2008) Bone remodeling, energy metabolism, and the molecular clock. Cell Metab 7: 7-10.

30. Rosen CJ (2012) Building bones by knocking down genes. Nat Med 18: 202 204.

31. Wang D, Miller SC, Shlyakhtenko LS, Portillo AM, Liu XM, et al. (2007) Osteotropic Peptide that differentiates functional domains of the skeleton. Bioconjug Chem 18: 1375-1378.

32. Zhang G, Guo B, Wu H, Tang T, Zhang BT, et al. (2012) A delivery system targeting bone formation surfaces to facilitate RNAi-based anabolic therapy. Nat Med 18: 307-314

33. Swami A, Reagan MR, Basto P, Mishima Y, Kamaly N, et al. (2014) Engineered nanomedicine for myeloma and bone microenvironment targeting. Proc Nat Acad Sci USA 111: 10287-10292.

34. Paley D, Catagni MA, Argnani F, Villa A, Benedetti GB, et al. (1989) Ilizarov treatment of tibial nonunions with bone loss. Clin Orthop Relat Res: 146-165.

35. Sato M, Ochi T, Nakase T, Hirota S, Kitamura Y, et al. (1999) Mechanica tension-stress induces expression of bone morphogenetic protein (BMP)2 and BMP-4, but not BMP-6, BMP-7, and GDF-5 mRNA, during distraction osteogenesis. J Bone Miner Res 14: 1084-1095.

36. Thies RS, Bauduy M, Ashton BA, Kurtzberg L, Wozney JM, et al. (1992) Recombinant human bone morphogenetic protein-2 induces osteoblastic differentiation in W-20-17 stromal cells. Endocrinology 130: 1318-1324.

37. Canalis E, Economides AN, Gazzerro E (2003) Bone morphogenetic proteins, their antagonists, and the skeleton. Endocr Rev 24: 218-235.

38. Harwood PJ, Giannoudis PV (2005) Application of bone morphogenetic proteins in orthopaedic practice: their efficacy and side effects. Expert Opin Drug Saf 4: 75-89.

39. Takuwa Y, Ohse C, Wang EA, Wozney JM, Yamashita K (1991) Bone morphogenetic protein-2 stimulates alkaline phosphatase activity and collagen synthesis in cultured osteoblastic cells, MC3T3-E1. Biochem Biophys Res Commun 174: 96-101.

40. Wang W, Mariani FV, Harland RM, Luo K (2000) Ski represses bone morphogenic protein signaling in Xenopus and mammalian cells. Proc Nat Acad Sci U S A 97: 14394-14399.

41. Wan DC, Pomerantz JH, Brunet LJ, Kim JB, Chou YF, et al. (2007) Noggin suppression enhances in vitro osteogenesis and accelerates in vivo bone formation. J Biol Chem 282: 26450-26459.

42. Rosen V (2006) BMP and BMP inhibitors in bone. Ann N Y Acad Sci 1068 $19-25$

43. Leboy PS (2006) Regulating bone growth and development with bone morphogenetic proteins. Ann N Y Acad Sci 1068: 14-18.

44. Urist MR (2009) The classic : a morphogenetic matrix for differentiation of bone tissue. Clin Orthop Relat Res 467: 3068-3070.

45. Urist MR, Strates BS (2009) The classic: Bone morphogenetic protein. Clin Orthop Relat Res 467: 3051-3062.

46. Brand RA1 (2009) Marshall R. Urist, 1914-2001. Clin Orthop Relat Res 467 3049-3050.

47. Urist MR (2002) Bone: formation by autoinduction. 1965. Clin Orthop Relat Res: 4-10.

48. Alam N, St-Arnaud R, Lauzier D, Rosen V, Hamdy RC (2009) Are endogenous BMPs necessary for bone healing during distraction osteogenesis? Clin Orthop Relat Res 467: 3190-3198.

49. Haidar ZS, Tabrizian M, Hamdy RC (2010) A hybrid rhOP-1 delivery system enhances new bone regeneration and consolidation in a rabbit model of distraction osteogenesis. Growth Factors 28: 44-55.

50. Hamdy RC, Amako M, Beckman L, Kawaguchi M, Rauch F, et al. (2003) Effects of osteogenic protein-1 on distraction osteogenesis in rabbits. Bone 33: 248 255.

51. Mandu-Hrit M, Seifert E, Kotsiopriftis M, Lauzier D, Haque T, et al. (2008) OP-1 injection increases VEGF expression but not angiogenesis in a rabbit model of distraction osteogenesis. Growth Factors 26: 143-151.

52. Rauch F, Lauzier D, Croteau S, Travers R, Glorieux FH, et al. (2000) Tempora and spatial expression of bone morphogenetic protein-2, -4 , and -7 during distraction osteogenesis in rabbits. Bone 27: 453-459.

53. Campisi P, Hamdy RC, Lauzier D, Amako M, Rauch F, et al. (2003) Expression of bone morphogenetic proteins during mandibular distraction osteogenesis. Plast Reconstr Surg 111: 201-208

54. Kloen P, Lauzier D, Hamdy RC (2012) Co-expression of BMPs and BMPinhibitors in human fractures and non-unions. Bone 51: 59-68.

55. Gazzerro E, Minetti C (2007) Potential drug targets within bone morphogenetic protein signaling pathways. Curr Opin Pharmacol 7: 325-333.

56. Groppe J, Greenwald J, Wiater E, Rodriguez-Leon J, Economides AN, et al (2003) Structural basis of BMP signaling inhibition by Noggin, a novel twelvemembered cystine knot protein. J Bone Joint Surg Am 85-85A Suppl 3: 52-8.

57. Devlin RD, Du Z, Pereira RC, Kimble RB, Economides AN, et al. (2003) Skeleta overexpression of noggin results in osteopenia and reduced bone formation. Endocrinology 144: 1972-1978.

58. Wu XB, Li Y, Schneider A, Yu W, Rajendren G, et al. (2003) Impaired osteoblastic differentiation, reduced bone formation, and severe osteoporosis in noggin-overexpressing mice. J Clin Invest 112: 924-934.

59. Iwata T, Morotome Y, Tanabe T, Fukae M, Ishikawa I, et al. (2002) Noggin blocks osteoinductive activity of porcine enamel extracts. J Dent Res 81: 387-391.

60. Edgar CM, Chakravarthy V, Barnes G, Kakar S, Gerstenfeld LC, et al. (2007) Autogenous regulation of a network of bone morphogenetic proteins (BMPs) mediates the osteogenic differentiation in murine marrow stromal cells. Bone 40: $1389-1398$

61. Tsialogiannis E, Polyzois I, Oak Tang Q, Pavlou G, Tsiridis E, et al. (2009) Targeting bone morphogenetic protein antagonists: in vitro and in vivo evidence of their role in bone metabolism. Expert Opin Ther Targets 13: 123-137.

62. Hannon GJ, Conklin DS (2004) RNA interference by short hairpin RNAs expressed in vertebrate cells. Methods Mol Biol 257: 255-266.

63. Moore CB, Guthrie EH, Huang MT, Taxman DJ (2010) Short hairpin RNA (shRNA): design, delivery, and assessment of gene knockdown. Methods Mol Biol 629: 141-158

64. Paddison PJ, Caudy AA, Sachidanandam R, Hannon GJ (2004) Short hairpin activated gene silencing in mammalian cells. Methods Mol Biol 265: 85-100.

65. Moffatt P, Gaumond MH, Salois P, Sellin K, Bessette MC, et al. (2008) Bril: a novel bone-specific modulator of mineralization. J Bone Miner Res 23: 14971508

66. Rawadi G, Vayssière B, Dunn F, Baron R, Roman-Roman S (2003) BMP-2 controls alkaline phosphatase expression and osteoblast mineralization by Wnt autocrine loop. J Bone Miner Res 18: 1842-1853.

67. Rungta RL, Choi HB, Lin PJ, Ko RW, Ashby D, et al. (2013) Lipid Nanoparticle Delivery of siRNA to Silence Neuronal Gene Expression in the Brain. Mol Ther Nucleic Acids 2: e136.

68. Zhigaltsev IV, Belliveau N, Hafez I, Leung AK, Huft J, et al. (2012) Bottom-up design and synthesis of limit size lipid nanoparticle systems with aqueous and triglyceride cores using millisecond microfluidic mixing. Langmuir 28: 36333640. 